\title{
Biological clocks and incremental growth line formation in dentine.
}

Amanda M. Papakyrikos ${ }^{1,2}$, Manish Arora ${ }^{3}$, Christine Austin $^{3}$, Julia C. Boughner ${ }^{4}$, Terence D. Capellini $^{5}$, Heather L. Dingwall ${ }^{5}$, Quentin Greba ${ }^{4}$, John G. Howland ${ }^{4}$, Akiko Kato ${ }^{5,6}$, Xiu-Ping Wang 7 , Tanya M. Smith $5,8,9^{*}$

${ }^{1}$ Department of Anthropology, Wellesley College, Wellesley, MA, USA

${ }^{2}$ Department of Developmental Biology, Stanford University School of Medicine, Stanford, CA, USA.

${ }^{3}$ Department of Environmental Medicine and Public Health, Icahn School of Medicine at Mount Sinai, New York, NY, USA.

${ }^{4}$ Department of Anatomy, Physiology and Pharmacology, College of Medicine, University of Saskatchewan, Saskatoon, SK, Canada

${ }^{5}$ Department of Human Evolutionary Biology, Harvard University, Cambridge, MA, USA

${ }^{6}$ Department of Oral Anatomy, School of Dentistry, Aichi Gakuin University, Nagoya, Japan

${ }^{7}$ Department of Developmental Biology, Harvard School of Dental Medicine, Boston, MA, USA ${ }^{8}$ Australian Research Centre for Human Evolution, Griffith University, Nathan, QLD, Australia ${ }^{9}$ Griffith Centre for Social and Cultural Research, Griffith University, Nathan, QLD, Australia

* Corresponding author: tanya.smith@griffith.edu.au 


\begin{abstract}
Dentine- and enamel-forming cells secrete matrix in consistent rhythmic phases, resulting in the formation of successive microscopic growth lines inside tooth crowns and roots. Experimental studies of various mammals have proven that these lines are laid down in subdaily, daily (circadian), and multidaily rhythms, but it is less clear how these rhythms are initiated and maintained. In 2001, researchers reported that lesioning the so-called master biological clock, the suprachiasmatic nucleus ( $\mathrm{SCN}$ ), halted daily line formation in rat dentine, while subdaily lines persisted. More recently, a key clock gene (Bmall) expressed in the SCN in a circadian manner was also found to be active in dentine- and enamel- secretory cells. In order to probe these potential neurological and local mechanisms for the production of rhythmic lines in teeth, we reexamined the role of the $\mathrm{SCN}$ in growth line formation in Wistar rats and investigated the presence of daily lines in Bmall knockout mice (Bmall ${ }^{-/-}$). In contrast to the results of the 2001 study, we found that both daily and subdaily growth lines persisted in rat dentine after complete or partial SCN lesion in the majority of individuals. In mice, after transfer into constant darkness, daily rhythms continued to manifest as incremental lines in the dentine of each Bmall genotype (wild-type, $\mathrm{Bmal}^{+/-}$, and $\mathrm{Bmall}^{-/}$). These results affirm that the manifestation of biological rhythms in teeth is a robust phenomenon, imply a more autonomous role of local biological clocks in tooth growth than previously suggested, and underscore the need to further elucidate tissue-specific circadian biology and its role in incremental line formation. Investigations of this nature will strengthen an invaluable system for determining growth rates and calendar ages from mammalian hard tissues, as well as documenting the early lives of fossil hominins and other primates.
\end{abstract}


Keywords: dental development, enamel, suprachiasmatic nucleus, biological rhythm, circadian rhythm, subdaily rhythm, ultradian rhythm, clock gene, Bmall, incremental feature

\section{Introduction}

Mammalian teeth preserve precise records of their development in the form of permanent incremental growth lines in dentine and enamel. These lines may show periodicities ranging from $\sim 5-12$ hours (subdaily lines), 24 hours (circadian/daily lines), to $2-12$ days (long-period lines) (Okada 1943; Bromage 1991; Ohtsuka and Shinoda 1995; Smith 2006; Kierdorf et al. 2013, 2019). Incremental lines can be counted to determine the age of juvenile primates at death, which may be accurate to within a few days or weeks of the actual age (Schwartz et al. 2006; Smith and Tafforeau 2008; Antoine et al. 2009; Smith 2013). Other broad applications include assessing diet transitions, estimating tooth eruption ages in deceased and extinct primates, and determining the age of developmental disruptions (e.g., Humphrey et al. 2008; Kelley and Schwartz 2010; Austin et al. 2013; Smith and Boesch 2015). Despite the increasing number of such studies by evolutionary anthropologists and oral biologists (reviewed in Smith 2008, 2013), fundamental questions remain about how incremental growth line timing is established and maintained, as well as why long-period line rhythmicity varies across primates (Dean 1995; Smith 2006, 2008; Bromage et al. 2009; Lacruz et al. 2012; Mahoney et al. 2018).

Many physiological and behavioral processes exhibit circadian rhythmicity, including hormone secretion, metabolism, growth, and sleep, which are controlled by internal biological clocks (reviewed in Mohawk et al. 2012; Neumann et al. 2019). The suprachiasmatic nucleus ( $\mathrm{SCN})$, a network of neurons located in the brain's anterior hypothalamus, is often referred to as the master biological clock. The SCN has the ability to adjust local biological clocks located in 
organs and cells throughout the body, and is cued by light levels absorbed by the retina (Ferguson and Cottrell 2002; Takahasi et al. 2008; Dibner et al. 2010). At a molecular level, biological clocks consist of a network of clock genes and transcription factors operating in a transcriptional-translational feedback loop (TTFL) (reviewed in Takahasi et al. 2008). The TTFL occurs within the cytoplasm and nucleus of individual cells within the SCN as well as in local biological clocks, lasts approximately 24 hours, and controls the transcription of a variety of evolutionarily conserved genes (Lee et al. 2011; Mohawk et al. 2012; Papagerakis et al. 2014; Zheng et al. 2014).

\section{Background}

\section{Experimental Research on Rodent Odonotogenetic Ryhthms}

Scholars have recently posited that incremental growth lines in dentine and enamel represent the output of biological clocks (e.g., Lacruz et al. 2012; Zheng et al. 2014). Support for this hypothesis comes from the work of Ohtsuka and Shinoda (1995) and Ohtsuka-Isoya et al. (2001). Ohtsuka and Shinoda (1995) injected 97 rat pups with nitrilotriacetato lead (Pb-NTA) at 4 - 7 day intervals to create known time-markers in the developing teeth. The rats were sacrificed 5 - 50 days after birth, and the dentine of the maxillary incisors was examined. The authors found evidence for two types of incremental lines each made up of a narrow (dark) band and a broader (light) band. Daily lines showed a spacing of $16-24 \mu \mathrm{m}$, while subdaily lines were spaced $6-8 \mu \mathrm{m}$ apart at an occurrence of 3 lines per day. These subdaily lines first appeared at the age of $7-10$ days, while daily lines began to appear by $12-15$ days of age. The authors reasoned that this timing was consistent with the development of many other physiological circadian rhythms. Importantly, they also stated that the initial appearance of daily 
lines in the $2^{\text {nd }}-3^{\text {rd }}$ week of postnatal life occurred when the rat brain was nearing maturation, pointing to its potential regulatory role for circadian rhythms in teeth.

Ohtsuka-Isoya and colleagues subsequently examined incremental growth line formation in the dentine of rats that were subject to surgical manipulation of the SCN (Ohtsuka-Isoya et al. 2001). They reported that in SCN-lesioned individuals, circadian locomotor behavior patterns ended after surgical manipulation, and that circadian lines permanently ceased formation as well. In some cases, their surgeries did not produce a complete lesion, resulting in either normal circadian line formation or a temporary loss of daily lines that was restored upon transfer to constant light conditions. Subdaily rhythms appeared to be more robust, as they were present in several individuals with either complete or partial SCN lesions. In one of three completely lesioned rats, subdaily line formation ceased until one week after the surgery, at which point 12hour lines were observed prior to sacrifice.

These results point to the importance of the SCN, as well as a local biological clock, in growth line formation - findings that are consistent with recent reports that components of the TTFL are expressed in a circadian pattern within isolated enamel-forming cells (ameloblasts) (Zheng et al. 2011; Lacruz et al. 2012). One such component is the Brain and Muscle ARNT-Like 1 (Bmall) gene, a transcription factor originally identified in the SCN (Bunger et al. 2000). The resulting protein BMAL1 shows a circadian rhythm in the SCN that peaks at night and decreases with exposure to light (Tamaru et al. 2000). BMAL1 works in concert with the transcription factor CLOCK to regulate the transcription of other key clock genes within a much larger complex network of molecules (Takahashi et al. 2008; Takahashi 2017).

\section{Biological Clock Mutants}


Pioneering experimental research in the 1990s showed that heritable mutations in the Clock gene lengthened the typical circadian periodicity of wheel-running activity in laboratorybred mice (Vitaterna et al. 1994). Another biological clock mutant mouse strain, Bmall (i.e., Bmall $^{-/}$), showed complete loss of circadian rhythms in locomotor activity and altered molecular transcription upon transfer to constant darkness (Bunger et al. 2000). While the $\mathrm{Bmal1}^{-/-}$mice lost circadian rhythmicity, heterozygotes maintained rhythmicity - showing no difference from wildtype mice. Importantly, the authors also analyzed the pattern of Bmall expression in the SCN, as well as in the liver, which is a well-known local biological clock. Bmall ${ }^{-/}$mice lost rhythmic expression of clock genes in both SCN and liver regions, suggesting global disruption of circadian biological clocks.

Zheng et al. (2011) reported that Bmall is expressed in dentine- and enamel-forming cells beginning at the bell stage of tooth formation at mouse embryonic day 17 (E17). Isolated and cultured enamel-forming cells show rhythmic activity of the main biological clock genes, including Bmall (Lacruz et al. 2012; Zheng et al. 2013). Moreover, Zheng et al. (2013) demonstrated that within enamel-forming cells in vivo, Bmall expression differed between the morning and evening - leading us to posit that $\mathrm{Bmall}^{-/-}$mice may be a useful model to explore the local mechanism that may lead to incremental growth line formation in teeth.

Here we revisited the roles of the SCN and putative local biological clocks during dentine formation. Using an experimental lead-labelling technique devised for marking time during dentine development, we first replicated the study of Ohtsuka-Isoya et al. (2001). We then applied this time-marking technique to study incremental line formation in the dentine of Bmal1 ${ }^{-}$ / mice. We tested the hypotheses that lesion of the SCN in rats would end daily incremental line formation, and that mice without Bmall expression would replicate this reported SCN-lesion 
phenotype by also failing to form daily incremental lines. To our knowledge, this represents the first attempt to assess the presence and periodicity of incremental lines in one of the most wellstudied clock mouse mutants, which is foundational to characterizing this important mutant as well as the precise clock mechanisms regulating the development of mammalian teeth.

\title{
Methods
}

\author{
Insert Table 1 here
}

\section{SCN Lesions}

Thirteen Wistar rats about 7 weeks old and weighing $176-200$ grams were purchased from Charles River Laboratories (Quebec, Canada). Rats were individually housed and acclimated to a normal 12:12 hour light/dark cycle for two weeks before the experiment, and food and water were provided ad libitum. In order to determine the role of the SCN in incremental line formation, rats were subjected to three treatments: non-surgical control, sham SCN surgery, and SCN lesion (Table 1). Following Ohtsuka and Shinoda (1995), all rats except the non-surgical control rat were injected subcutaneously with lead nitrilotriacetic acid (Pb-NTA, $2 \mathrm{mg} / \mathrm{kg}, \mathrm{pH} \sim 7.4$ ) to create known time-markers in the developing incisors. Three injections were given at approximately the same time five days prior to surgery, on the day of the surgery, and seven days after surgery, followed by sacrifice four days later.

On the day of the surgery, six rats underwent a SCN lesion procedure consistent with the protocol of Ohtsuka-Isoya et al. (2001). Rats were anesethized with isoflurane, placed in a stereotaxic frame, and the dorsal surface of the skull was exposed and cleaned. The SCN was targeted bilaterally $(\mathrm{AP}-0.7 \mathrm{~mm}, \mathrm{ML} \pm 0.2 \mathrm{~mm}, \mathrm{DV}-9.4 \mathrm{~mm}$, all coordinates from bregma 
with level skull) with an insulated nichrome wire (Leico Industries Inc., New York). Insulation $(0.5 \mathrm{~mm})$ was scraped from the electrode tip prior to surgery. Once the electrode was lowered into position, anodal current $(0.3 \mathrm{~mA})$ was passed through the wire for 3 minutes. The electrode was then slowly raised out of the brain and the procedure was repeated for the other hemisphere. The resulting holes in the skull were then filled with bone wax, and the skin above the skull was sutured. Six additional rats underwent a sham surgery wherein no current was passed through the wire used to destroy the brain tissue in the other six rats. In these sham control rats, the wire was inserted just dorsal to the SCN so as not to damage this structure.

Following surgery, the 12 rats were transferred to constant light conditions to eliminate external circadian cues. Seven days after surgery, rats were given the third and final injection of $\mathrm{Pb}-\mathrm{NTA}$. Four days after this final injection, the rats were sacrificed by perfusion through the left cardiac ventricle with a fixative solution containing $2.5 \%$ glutaraldehyde and $2 \%$ paraformaldehyde in $0.1 \mathrm{M}$ phosphate buffer ( $\mathrm{pH}$ 7.2) under excessive pentobarbital sodium anesthesia. Following sacrifice, brains, mandibles, and maxillary bones were dissected out and fixed by immersion in the same fixative solution as above for 48 hours. Brains were sectioned to verify the position of lesions (Supplemental Figure 1). These experiments were approved by the Animal Research Ethics Board at the University of Saskatchewan (Boughner: 20110008).

Insert Table 2 here

\section{Biological Clock Knockout Mice}

Bmal1 $^{+/-}$mice on a C57BL/6 background (stock \#009100) were purchased from Jackson Laboratory and crossed to produce $\mathrm{Bmall}^{-/-}$mice and control genotypes. PCR-based genotyping 
of the offspring was performed according to the methods of Bunger et al. (2000). Mice were housed in 12:12 hour light/dark conditions, provided food and water ad libitum, and acclimated to the experimental space for $1-2$ weeks before beginning the experiment. In order to assess the differences in incremental line formation between $\mathrm{Bmall}^{-/}, \mathrm{Bmal}^{+/}$, and wild-type mice, five individuals were given $3-4$ intraperitoneal injections of Pb-NTA at $4-7$ day intervals (Table 2). Injections were given at approximately the same time every day. After the second injection, mice were transferred from a 12:12 light/dark cycle to constant darkness until sacrifice. Three to four days after the last injection, mice were sacrificed by $\mathrm{CO}_{2}$ overdose and cervical dislocation. The mandibles and maxillae were dissected out and fixed in $2.5 \%$ glutaraldehyde at $4{ }^{\circ} \mathrm{C}$ for 48 hours. These experimental procedures were approved by the Institutional Animal Care and Use Committee at Harvard University (Capellini: 13-04-161-2).

Insert Figure 1 here

\section{Incremental Line and Lead Label Visualization}

To first determine whether or not SCN lesion affected the internal morphology of the rat incisors, we prepared hard tissue sections from each of the three experimental groups (lesion, sham surgery, non-surgical control). Samples for hard-tissue sectioning were soaked in methylacrylate (MMA) monomer for three days and then embedded in catalyzed MMA in a $48^{\circ} \mathrm{C}$ oven for 3 days. Embedded incisors were cut transversely (Figure 1) using an Isomet low speed diamond saw, and 0.6-1.0 mm thick sections were removed. These sections were polished with $1 \mu \mathrm{m}$ alumina suspension, glued to a microscope slide with UV curing resin, ground to a final thickness of 30-100 $\mu \mathrm{m}$, polished, and cover slipped using DPX mounting media. 
In order to visualize the lead labels and incremental lines together, rat hemi-mandibles and hemi-maxillae were decalcified for $2-5$ weeks in $0.5 \mathrm{M}$ ethylenediaminetetraacetic acid (EDTA) and 5\% sodium sulfide following the protocol of Ohtsuka and Shinoda (1995). The solution was changed daily to speed up decalcification. Once decalcified, the samples were rinsed with running water and phosphate buffered saline (PBS), and then washed in 5\% sucrose before refrigeration in a $30 \%$ sucrose solution overnight. Samples were then transferred to a 1:1 ratio of $30 \%$ sucrose and optimal cutting temperature compound (OCT) the following day, and stored overnight in a refrigerator at $4^{\circ} \mathrm{C}$. Next, the samples were embedded in OCT and frozen at $-80^{\circ} \mathrm{C}$ overnight. Frozen samples were cut transversely using a cryostat in order to produce 20 $\mu \mathrm{m}$ thick sections, which were kept at $-20^{\circ} \mathrm{C}$ or $4^{\circ} \mathrm{C}$ until staining. The sections were then dried and rinsed in distilled $\mathrm{H}_{2} \mathrm{O}$ to remove excess OCT, and treated with $0.1 \%$ gold chloride for 20 minutes, followed by $5 \%$ sodium thiosulfate for 20 minutes. Finally, sections were stained with either Carazzi's or Harris's hematoxylin to enhance the lead labels and incremental growth lines before mounting with AquaMount.

Histological sections were imaged under transmitted light with varying degrees of polarization using an Olympus BX51 transmitted light microscope (Figure 1). A MicroPublisher 5.0 digital camera and Olympus MicroSuite imaging and analysis software were used to photograph and quantify the spacing between lead labels and incremental lines. A.M.P. and T.M.S. independently verified the presence of incremental lines in rodents from each treatment group and condition.

Insert Figures 2 and 3 here 


\section{Results}

\section{Validation of Experimental Manipulation}

Because Appleton (1991) and Hillson (2014) have expressed doubt about the retention of lead in the experimental lines produced by the labeling protocol of Ohtsuka and Shinoda (1995), we performed laser ablation-inductively coupled plasma mass spectrometry following the protocol outlined by Austin et al. (2013) on an embedded longitudinal section of a maxillary incisor from a wild-type mouse (C57BL/6J background). The mouse, part of an initial proof-ofconcept study, had been injected subcutaneously 3 times at 5-day intervals with Pb-NTA as described above. The presence of three discrete bands of elevated lead values confirmed that this labelling approach deposits lead in mineralizing tissues, including the maxillary bone (Figure 2).

Visual inspection of incisors sectioned in the same plane and position from the nonsurgical control, sham surgery, and SCN lesioned rats verified that these teeth were structurally sound and that neither hard tissue processing nor demineralization affected the ability to detect incremental growth lines (Figure 3). Further, no obvious pathologies or structural deviations were observed in the sections, although an accentuated line was often observed following the surgical procedure. Notably, incremental growth lines were often clearer in mineralized sections than in demineralized sections. This enhanced clarity may relate to the greater thickness of the mineralized sections, leading to optical superimposition of increments located above and below the focal plane. Importantly, we could not quantify the periodicity of incremental lines in mineralized sections because the lead lines that serve as time markers are not visible prior to decalcification and staining. This demineralization requirement also prevented analysis of incremental lines in enamel. 
Insert Figure 4 here

\section{SCN Lesions}

\section{$\underline{\text { Non-surgical control rat }}$}

Incremental growth lines similar to the daily and subdaily lines illustrated by OhtsukaIsoya et al. (2001) were visible in the non-surgical control. Thick, widely-spaced dark stained hematoxylin bands were seen in the outer portion of the incisor; these grew fainter towards the inner portion (Figure 4A). Distinct closely-spaced incremental lines were apparent in the lingual portion of the incisor near the pulp chamber. It was not possible to quantify the temporal nature of these lines as this individual had not been injected with lead labels.

\section{$\underline{\text { Sham surgery rats }}$}

In all sham surgery rats, daily growth lines were observed between lead labels 1 and 2 , demonstrating that daily lines were present prior to the sham surgery (performed on the day of the $2^{\text {nd }}$ injection). Daily lines were detected between labels 2 and 3 in the majority of the rats subject to a sham surgery (Figure 4C), and were clearly seen between the $3^{\text {rd }}$ label and the day of sacrifice in half of these rats, while in the the other half the daily lines were more ambiguous. Subdaily lines with a repeat interval of less than 24 hours were seen in all experimental intervals, although these subdaily lines were not as well defined prior to the sham surgery (Figure 4B).

\section{Insert Figure 5 here}

\section{$\underline{\text { Rats with unilateral or posterior lesions }}$}


Post hoc neuroanatomical analysis confirmed that three of the six intended SCN lesion surgeries were partial; two rats received a unilateral lesion and one rat received a lesion of the hypothalamus posterior to the SCN. In both rats with the unilateral lesions, daily and subdaily lines were present before and after the surgery. The rat with the posterior lesion showed clear daily lines pre-surgery, and strong subdaily lines with a repeat intervals of $\sim 2$ and $\sim 3$ lines per day post-surgery (Figure 5). Other sections from this individual confirmed the presence of daily and subdaily lines within each interval prior to sacrifice.

Insert Figure 6 here

\title{
Complete SCN lesion rats
}

Post hoc neuroanatomical analysis confirmed that three rats received complete lesions of their SCNs. Daily growth lines were present before the surgery; these lines were similar in clarity and spacing to the lines seen in all other treatment groups. After the SCN lesion, daily lines were fainter (Figure 6), yet still seen in two of these three rats. Subdaily lines with a repeat interval of approximately 12 hours were present in all three rats before and after complete surgical lesion of the SCN (Figure 7).

\author{
Insert Figure 7 here
}

\section{Biological Clock Knockout Mice}

Four daily lines were detected between all labels in the wild-type mouse, and these lines remained clearly visible after transfer to constant darkness following the $2^{\text {nd }}$ injection (Figure 
8A). In some wild-type mouse sections, faint subdaily lines were apparent between intervals. Daily incremental lines were also detected in both $\mathrm{Bmal}^{+/-}$mice (Fig. 8B) and both $\mathrm{Bmall}^{-/-}$mice (Fig. 8C), and these lines persisted after transfer to constant darkness. Subdaily lines were evident in the two Bmall $^{-/-}$mice, although it was not possible to determine their repeat interval due to variable clarity of these lines in the incisor tissue sections.

\author{
Insert Figure 8 here
}

\title{
Discussion
}

\section{Presence of Rhythmic Growth Increments in Teeth}

Experimental studies of rodents and primates conclusively demonstrate that daily growth lines form as a result of the secretory activity of dentine- and enamel-forming cells (odontoblasts and ameloblasts, respectively) (e.g., Okada 1943; Shinoda 1984; Bromage 1991; Ohtsuka and Shinoda 1995; Smith 2006). Our results from both rats and mice are consistent with these previous findings. Early work implicated physiological rhythms in $\mathrm{pH}$, calcium/phosphate levels, and collagen composition/orientation as proximate causes of growth lines (Okada 1943; Shinoda 1984; Boyde 1989). Ohtsuka et al. (1998) conducted an elegant experimental study of rat dentine with hydroxyproline, a labeled version of the essential amino acid proline used in collagen synthesis. Her team found that the pair of alternating dark and light bands that make up each daily incremental line relate to regions of collagen that are rapidly secreted during the environmentally light period (diurnal resting time) and more slowly during the dark period (nocturnal active time). Moreover the rhythmic darkly-stained hematoxylin bands - reflecting areas of increased acidity - also indicated circadian shifts in $\mathrm{pH}$. They suggested that rapid 
diurnal collagen synthesis may be followed $8-12$ hours later by a peak in dentine mineralization during the nocturnal period - implying different rhythmic processes over the course of a single day of dentine formation.

We also found incremental lines with repeat intervals of $\sim 2-3$ lines per day in rats (as well as in some mice), which were typically clearest after the SCN surgery $\left(2^{\text {nd }}\right.$ lead label: Figure $4 \mathrm{~B}, 5)$. This is consistent with recent work suggesting that after modification the SCN, subdaily rhythms persist and may become more robust (reviewed in Wu et al. 2018). Subdaily lines were also very clear in the middle and last-formed portions of sections from the control and sham rat incisors, supporting the natural expression of these subdaily lines. Others have experimentally demonstrated the presence of subdaily lines with a periodicity of $\sim 2$ or $\sim 3$ lines per day in rabbit and primate hard tissues (reviewed in Ohtsuka and Shinoda 1995; Smith 2006). Even more frequent rhythmic lines on the order of 5 lines per day have been demonstrated in sheep and pig enamel (Kierdorf et al. 2013, 2019).

Ohtsuka and Shinoda (1995) suggested that subdaily dentine lines could result from initial rhythms in dentine secretion, or subsequent dentine mineralization rhythms, but that further information was needed to distinguish between these two possibilities. We concur with this, particularly given the difficulty of visualizing subdaily lines consistently (discussed and illustrated for primate enamel in Smith 2006). For example, our rodent sections showed subdaily lines more commonly in the last $1-2$ weeks of dentine formation relative to the preceding week or more. We question whether EDTA penetrance may be more limited in the deepest regions, leading to differences in the degree of demineralization that could impact the appearance of growth lines. Determining the proximate cause of subdaily rhythms in teeth is a considerable challenge given the limitations of current time-marking and visualization methods. Experimental 
studies that are able to probe both secretion and mineralization processes in tandem, as well as future improvements in synchrotron virtual histology (e.g., Tafforeau et al. 2007; Tafforeau and Smith 2008), may aid in this endeavor.

\section{SCN Lesions}

Our results on experimentally manipulated rats are only partially consistent with previous studies. Measurements between lead labels revealed that dentine secretion proceeded at $16.7-$ $24.1 \mu \mathrm{m} /$ day, nearly identical to the rates of Ohtsuka and Shinoda (1995), suggesting that our time-marking procedure produced comparable results. Yet our observations of the three different manipulations of the hypothalamus differed from those of Ohtsuka-Isoya et al. (2001). In both instances of unilateral SCN lesion in our study, daily growth lines were present before and after the surgery. Ohtsuka-Isoya et al. (2001) reported that two unilaterally-lesioned rats showed consistent daily lines while two other unilaterally-lesioned rats showed some disruption. One rat in our study and one rat in that of Ohtsuka-Isoya et al. (2001) also had damage in the paraventricular region posterior to the $\mathrm{SCN}$. For this latter rat, growth lines were reported to be absent following surgery - yet for the rat in our study, daily lines were visible during the entire period of incisor growth prior to sacrifice. Also, in this same rat we found distinct subdaily lines with a repeat interval of $\sim 2$ and $\sim 3$ lines per day immediately after the SCN surgery ( $2^{\text {nd }}$ label in Figure 5). In contrast, Ohtsuka-Isoya et al. (2001) reported that subdaily lines were not evident until 7 days after surgery.

Importantly, our observations in rats with complete SCN lesions also differ from those of Ohtsuka-Isoya et al. (2001), who reported that daily growth lines ceased forming immediately after complete SCN lesion in all three rats. Our three fully-lesioned rats showed daily lines in the 
week following the surgery; two of these continued until sacrifice, four days after the final label (11 days post-surgery). While daily growth lines were difficult to visualize after the surgery, they could often be seen when a section was taken slightly out of focus (Figure 6). This improved resolution may be due to increases in the optical superimposition of incremental lines caused by moving the focal plane just above or below the thin slice of tissue.

In one of Ohtsuka-Isoya et al. (2001)'s three complete SCN-lesioned rats, subdaily growth lines ( $\sim 2$ lines per day) appeared a week after surgery, while in the other two rats, subdaily growth lines appeared immediately after the operation. In contrast, we found that subdaily lines were evident in all three of our rats both prior to and immediately after surgery. Considered together, these results suggest that a functional SCN is not necessary for the formation of daily and subdaily growth lines, and that there is variation in the clarity of these features within and between studies. It is likely that the disparate findings of the current study and those of Ohtsuka-Isoya et al. (2001) are at least partially due to subtle differences in processing, sectioning, and/or imaging. It was evident to us that incremental line clarity varied among individuals in the same treatment group, among incisors from the same individual, and among serial sections of the same incisor, potentially leading to misleading results if only a small number of tissue sections are examined.

Ohtsuka and Shinoda (1995) postulated that daily lines do not appears in rats until $2-3$ weeks of age, which they linked to the maturation of the brain (including the SCN) at 3 weeks old. However, recent studies indicate that structural maturation of the brain of rats may continue until about two months of age (Semple et al. 2013), while the SCN matures 10 days after birth (Landgraf et al. 2010). Intriguingly, rhythmic circadian clock gene activity begins in the SCN around embryonic day 19 (E19) in rats and E17 in mice. While the rats and mice included in this 
study were too old to explore incremental line formation at such young ages, prenatal and perinatal daily lines have been documented in non-human primates and humans (e.g., Smith 2006; Birch and Dean 2014) - long before the maturation of the primate brain or the establishment of circadian rhythms in some activity cycles (Hellbrügee 1960). Additional research is needed to elucidate whether the initiation of incremental growth lines requires a fully mature SCN, the expression of rhythmic clock genes, or another type of developmental cue.

\section{Biological Clock Knockout Mice}

In order to explore a hypothesized molecular control mechanism for rhythmic growth line formation, we applied the same time-marking method to a mouse mutant with a global disruption in a transcription factor (BMAL1) implicated in the circadian biological clock (Bunger et al. 2000). Previous investigations of Bmall expression in dentine- and enamel- secretory cells used wild-type mouse strains (Zheng et al. 2011, 2013) or isolated enamel-secretory cells (Lacruz et al. 2012; Zheng et al. 2013). Zheng et al. (2013) reported that Bmall expression differed between the morning and evening - similar to the rhythmic pattern of proline incorporation during collagen synthesis documented by Ohtsuka et al. (1998). However it has been unclear whether rhythmic BMAL1 production influences the formation of daily lines in dentine or enamel.

Here we demonstrated that in homozygous $\left(\mathrm{Bmall}^{-/-}\right)$mice, daily lines in dentine are present, albeit sometimes fainter than those in wild-type and Bmal1 ${ }^{+/-}$mice. Our results suggest that Bmall has little to no role, or - perhaps more likely - a redundant role, in the regulation of rhythmic dentine secretion in rodent tooth. This is consistent with a recent report of circadian oscillations in both RNA and protein synthesis in skin fibroblast and liver tissue from Bmal1 $^{-/-}$ 
mice (Ray et al. 2020). Thus it appears that daily growth lines produced during dentine formation require neither a functional SCN nor Bmall gene.

Intriguingly, Bunger et al. (2000) reported that residual low amplitude subdaily rhythms $\left(5-12\right.$ hours) persisted in the locomotor activity of Bmall $^{-/-}$mice housed in constant darkness, and that these rhythms were not statistically different from those of wild-type and $\mathrm{Bmal}^{+/-}$mice. They reviewed evidence for similar subdaily rhythms in other biological clock mutant mice and animals subjected to SCN manipulation, and concluded that subdaily rhythms occur independent of circadian oscillators. Writing prior to our contemporary understanding of clock genes, Newman and Poole $(1974,1993)$ originally suggested that long-period ( $>1$ day) incremental growth lines in teeth may be the result of multiple shorter-period rhythms regularly intersecting. Recent work in molecular biology is consistent with this hypothesis, as various transcription factors controlled by clock genes show a wide range of cyclical expression intervals ranging from minutes to hours (Korenic et al. 2014). Given the presence of daily lines in the dentine of $\mathrm{SCN}-$ lesioned rodents and $\mathrm{Bmall}^{-/}$mice, and the large range of subdaily rhythms reported for mammals, it is also possible that a phenomenon of intersecting subdaily rhythms underpins the production of daily lines in teeth. For example, multiple 12- or 8-hour rhythms will overlap every 2 or 3 cycles, respectively, which would lead to an incidental 24-hour rhythm.

Our exploration of daily and subdaily increments in rodent dentine did not reveal the presence of rhythms in excess of one day, which are well known from the dentine and enamel of primates and other mammals (e.g., Smith 2006; Tafforeau et al. 2007; Bromage et al. 2009; Kierdorf et al. 2019). The repeat interval of these long-period lines is best assessed through experimental labeling, as it can be difficult to distinguish subdaily and daily increments from one 
another - leading to errors in the determination of long-period line repeat intervals, daily secretion rates, and crown formation times (Smith 2006; Kierdorf et al. 2019).

\section{Conclusions}

Experimental studies have established the rhythmic cellular formation of daily growth lines in mammalian dentine, which continues under constant lighting conditions (Shinoda, 1984; Ohtsuka-Isoya et al. 2001; this study), as well as throughout extreme physiological challenges (e.g., Schwartz et al. 2006; Smith 2013; Austin et al. 2016). However the ultimate control mechanism responsible for the initiation and regulation of these lines remains elusive, as dentineforming cells may continue to produce daily growth lines after the functional loss of the master biological clock in the SCN. It has become clear from experimental work, including sequencing projects, that the regulation of both master and peripheral clocks is hierarchical, multi-factorial and more complex than initially understood, with inputs from the environment as well as from within the organism itself (reviewed in Takahashi 2017). Future studies may explore the importance of other regions of the hypothalamus for the regulation of growth increments, as recent work in mice has shown that cultured slices of neural regions adjacent to the SCN generate subdaily rhythms that are transmitted to the SCN (Wu et al. 2018).

We have also shown that the lack a local rhythmically-produced transcription factor, BMAL1, did not lead to the cessation of daily growth increments in Bmall $^{-/}$mice after transfer to constant darkness. Other clock genes are known to be active during tooth formation, including Clock, Per1, and Per2 (Zheng et al. 2011; Lacruz et al. 2012), which could be similarly explored in mutant mice strains. Given the functional relationship between BMAL1 and CLOCK, concurrent alterations in both of these genes may have a greater influence on circadian 
increments in mammalian teeth. Advances in gene editing technologies are permitting novel insights into circadian biology through targeted gene ablations (Tsuchiya et al. 2016). Finally, others have hypothesized that the central nervous system may control incremental growth line formation through rhythmic melatonin secretion (Kumasaka et al. 2010; Ji et al. 2011; Tao et al. 2016) - an intriguing possibility that remains to be tested.

\section{Acknowledgements}

Rachel Carmody, Lenia Constantinou, Louise Darling, Lawrence Flynn, Daniel Green, Meg Jarvi, Meg Lynch, Catherine MacGillivray, Michele Moroz, Ping Shao, Arlene Santos-Diaz, Paul Tafforeau, Christine Tafoya, Nancy Tang, Adam Van Arsdale, Matthew Warman, Charles Weitz, Yong Wen, and Irina Zhdanova provided research assistance and helpful discussions. Funding was provided by the American School of Prehistoric Research, Harvard University, Harvard School of Dental Medicine, University of Saskatchewan College of Medicine, the Natural Sciences and Engineering Research Council of Canada (Discovery Grant 2016-05177 to J.C.B.) and the Howard Hughes Medical Institute. A.M.P was supported by a Genetech Fellowship and a Wellesley Summer Research Grant.

\section{Author Contributions}

Study design: AMP, JCB, TDC, TMS; Substantial logistical facilitation and/or analytical support: MA, CA, JCB, TDC, HLD, QG, JGH, AK, X-PW, YW, TMS; Data acquisition and interpretation: AMP, QG, JGH, TMS; Drafting of manuscript: AMP, JCB, TMS; 
Revision and approval of the manuscript: all co-authors. The data that support the findings of this study are available from the corresponding author upon reasonable request.

\section{References}

Antoine D, Hillson D, Dean MC (2009) The developmental clock of dental enamel: a test for the periodicity of prism cross-striations in modern humans and an evaluation of the most likely sources of error in histological studies of this kind. J Anat 214, 45-55.

Appleton J (1991) The effect of lead acetate on dentine formation in the rat. Arch Oral Biol 36, $377-382$.

Austin C, Smith TM, Bradman A et al. (2013) Barium distributions in teeth reveal early life dietary transitions in primates. Nature 498, 216-219.

Austin C, Smith TM, Farahani RMZ et al. (2016) Uncovering system-specific stress signatures in primate teeth with multimodal imaging. Sci Rep 6, 18802.

Birch W, Dean MC (2014) A method of calculating human deciduous crown formation times and of estimating the chronological ages of stressful events occurring during deciduous enamel formation. Journal of Forensic and Legal Medicine 22, 127-144.

Boyde A (1989) Enamel. In Handbook of Microscopic Anatomy, Vol. V/6: Teeth (eds Oksche A, Vollrath L), pp. 309-473. Berlin: Springer.

Bromage TG (1991) Enamel incremental periodicity in the pigtailed macaque: a polychrome fluorescent labeling study of dental hard tissues. Am J Phys Anthropol 86, 205-214. 
Bromage TG, Lacruz RS, Hogg R, et al. (2009) Lamellar bone is an incremental tissue reconciling enamel rhythms, body size, and organismal life history. Calcif Tissue Int 84, 388404.

Bunger MK, Wilsbacher LD, Moran SM, et al. (2000) Mop3 is an essential component of the master circadian pacemaker in mammals. Cell 103, 1009-1017.

Dean MC (1995) The nature and periodicity of incremental lines in primate dentine and their relationship to periradicular bands in $\mathrm{OH} 16$ (Homo habilis). In: Moggi-Cecchi, J. (Ed.), Aspects of Dental Biology: Paleontology, Anthropology and Evolution. International Institute for the Study of Man, Florence, pp. 239-265.

Dibner C, Schibler U, Albrecht U (2010) The mammalian circadian timing system: organization and coordination of central and peripheral clocks. Annu Rev Physiol 72, 517-549.

Ferguson AV, Cottrell GT (2002) Time to consider new brain clock signals. TRENDS in Endocrinology \& Metabolism 13, 276-277.

Hellbrügee T (1960) The development of circadian rhythms in infants. Cold Spring Harbor Symposium on Quantitative Biology 25, 311-323.

Hillson S (2014) Tooth Development in Human Evolution and Bioarchaeology. Cambridge: Cambridge University Press.

Humphrey LT, Dean MC, Jeffries TE et al. (2008) Unlocking evidence of early diet from tooth enamel. Proc Natl Acad Sci USA 105, 6834-6839.

Ji F, Tao J, Jia W, Shen G (2011). Growing teeth in the dark: circadian rhythmic tooth growth regulated by melatonin? Dental Hypotheses 2, 99-104. 
Kelley J, Schwartz G. (2010). Dental development and life history in living African and Asian apes. Proc Natl Acad Sci USA 107, 1037-1040.

Kierdorf H, Kierdorf U, Frölich K, Witzel C (2013) Lines of evidence - incremental markings in molar enamel of Soay sheep as revealed by a fluorochrome labeling and backscattered electron imaging study. PLoS One 8, e74597.

Kierdorf H, Breuer F, Witzel C, Kierdorf U (2019) Pig enamel revisited - Incremental markings in enamel of wild boars and domestic pigs. J Structural Biology 205: 48-59.

Korencic A, Kosir R, Bordyugov G, et al. (2014) Timing of circadian genes in mammalian tissues. Sci Reps 4, 5782.

Kumasaka S, Shimozuma M, Kawamoto T, et al. (2010) Possible involvement of melatonin in tooth development: expression of melatonin 1a receptor in human and mouse tooth germs. Histochem Cell Biol 133, 577-584.

Lacruz RS, Hacia JG, Bromage TG, et al. (2012). The circadian clock modulates enamel development. J Biol Rhythms, 27, 237-245.

Landgraf D, Koch CE, Oster H (2014). Embryonic development of circadian clocks in the mammalian suprachiasmatic nuclei. Frontiers in Neuroanatomy 8 Article 143

Lee HM, Chen R, Kim H, Etchegaray JP, Weaver DR, Lee C (2011). The period of the circadian oscillator is primarily determined by the balance between casein kinase 1 and protein phosphatase 1. Proc Natl Acad Sci USA 108, 16451-16456.

Mahoney P, Miszkiewicz JJ, Chapple S. et al. (2018) The biorhythm of human skeletal growth. J Anat 232, 26-38 
Mohawk JA, Green CB, Takahashi JS (2012) Central and peripheral circadian clocks in mammals. Annu Rev Neurosci 35, 445-462.

Neumann A-M, Schmidt CX, Brockmann RM, Oster H (2019) Circadian regulation of endocrine systems. Autonomic Neuroscience: Basic and Clinical 216, 1-8.

Newman HN, Poole DFG (1974) Observations with scanning and transmission electron microscopy on the structure of human surface enamel. Arch Oral Biol 19, 1135-1143.

Newman HN, Poole DFG (1993) Dental enamel growth. J R Soc Med 86, 61.

Ohtsuka M, Shinoda H (1995) Ontogeny of circadian dentinogenesis in the rat incisor. Arch Oral Biol 40, 481-485.

Ohtsuka M, Saekil S, Igarashil K, Shinoda H (1998) Circadian rhythms in the incorporation and secretion of $3 \mathrm{H}$-proline by odontoblasts in relation to incremental lines in rat dentin. J Dent Res $77,1889-1895$

Ohtsuka-Isoya M, Hayashi H, Shinoda H (2001) Effect of suprachiasmatic nucleus lesion on circadian dentin increment in rats. Am J Physiol Reg Comp Physiol 280, R1364-R1370.

Okada M (1943) Hard tissues of animal body: highly interesting details of Nippon studies in periodic patterns of hard tissues are described. In The Shanghai Evening Post Special Edition, Health, Recreation and Medical Progress, pp. 15-31.

Papagerakis S, Zheng L, Schnell S, et al. (2014) The circadian clock in oral health and diseases. J Dent Res 93, 27-35. 
Paxinos G, Watson C (2006) The Rat Brain in Stereotaxic Coordinates, 6th ed., New York: Academic Press.

Ray S, Valekunja UK, Stangherlin A, et al. (2020) Circadian rhythms in the absence of the clock gene Bmall. Science 367, 800-806.

Schwartz GT, Reid DJ, Dean MC et al. (2006) A faithful record of stressful life events preserved in the dental developmental record of a juvenile gorilla. Int J Primatol 27, 1201-1219.

Semple BD, Blomgren K, Gimlin K, Ferriero DM, Noble-Haeusslein LJ (2013) Brain development in rodents and humans: Identifying benchmarks of maturation and vulnerability to injury across species. Progress in Neurobiology 106-107, 1-16.

Shinoda H (1984) Faithful records of biological rhythms in dental hard tissues. Chem Today 9 , 34-40.

Smith TM. (2006) Experimental determination of the periodicity of incremental features in enamel. J Anat 208, 99-113.

Smith TM (2008) Incremental dental development: methods and applications in hominoid evolutionary studies. J Hum Evol 54, 205-224.

Smith TM (2013) Teeth and human life-history evolution. Annu Rev Anthropol 42, 191-208.

Smith TM, Boesch C (2015) Developmental defects in the teeth of three wild chimpanzees from the Taï Forest. Am J Phys Anthropol 157, 556-570.

Smith TM, Tafforeau P. (2008) New visions of dental tissue research: tooth development, chemistry, and structure. Evol Anthropol 17, 213-226. 
Tafforeau P, Bentaleb I, Jaeger J-J, Martin C. (2007) Nature of laminations and mineralization in rhinoceros enamel using histology and X-ray synchrotron microtomography: Potential implications for palaeoenvironmental isotopic studies. Palaeogeography, Palaeoclimatology, Palaeoecology 246, 206-227.

Tafforeau P, Smith TM (2008) Nondestructive imaging of hominoid dental microstructure using phase contrast X-ray synchrotron microtomography. J Hum Evol 54, 272-278.

Tamaru T, Isojima Y, Yamada T, Okada M, Nagai K, Takamatsu K (2000) Light and glutamateinduced degradation of the circadian oscillating protein BMAL1 during the mammalian clock resetting. The Journal of Neuroscience 20, 7525-7530.

Takahashi JS (2017) Transcriptional architecture of the mammalian circadian clock. Nature Reviews Genetics 18, 164-179.

Takahashi JS, Hong H-K, Ko CH, McDearmon EL (2008) The genetics of mammalian circadian order and disorder: implications for physiology and disease. Nature Reviews Genetics 9, 764775.

Tao J, Zhai Y, Park H, et al. (2016) Circadian rhythm regulates development of enamel in mouse mandibular first molar. PLoS ONE 11, e0159946.

Tsuchiya Y, Umemura Y, Minami Y, et al. (2016) Effect of multiple clock gene ablations on the circadian period length and temperature compensation in mammalian cells. J Biol Rhythms 31, $48-56$.

Vitaterna MH, King DP, Chang A-M., et al. (1994) Mutagenesis and mapping of a mouse gene, Clock, essential for circadian behavior. Science 264, 719-725. 
Wu Yu-Er, Enokid R, Odad Y, Huang Z-L, Honma K, Honmad S. (2018) Ultradian calcium rhythms in the paraventricular nucleus and subparaventricular zone in the hypothalamus. Proc Natl Acad Sci USA 115, E9469-E9478.

Zheng L, Papagerakis S, Schnell SD, Hoogerwerf WA, Papagerakis P (2011) Expression of clock proteins in developing tooth. Gene Expr Patterns 11, 202-206.

Zheng L, Seon YJ, Mourão MA, et al. (2013) Circadian rhythms regulate amelogenesis. Bone 55, $158-165$.

Zheng L, Ehardt L, McAlpin B, et al. (2014) The tick tock of odontogenesis. Exp Cell Res 325, 83-89. 
Table 1. Experimental treatment groups for the rat SCN lesion study.

Group $\quad$ n $\quad$ Treatment

Control

1

No lead injections, no surgery, no light manipulations

Sham

6

Lead injections, sham surgery, light manipulations

SCN lesion

6

Lead injections, surgery, light manipulations

3 complete bilateral lesions

2 partial unilateral lesions

1 posterior hypothalmus lesion 
Table 2. Treatment for the Bmall mice experiment.

\begin{tabular}{|c|c|c|c|c|}
\hline Experiment & Genotypes & Event & Age (days) & Interval (days) \\
\hline \multirow[t]{5}{*}{ Replicate 1} & WT, Het, Homo & Injection 1 & 59 & - \\
\hline & & Injection $2^{+}$ & 63 & 4 \\
\hline & & Injection 3 & 67 & 4 \\
\hline & & Injection 4 & 71 & 4 \\
\hline & & Sacrifice & 75 & 4 \\
\hline \multirow[t]{4}{*}{ Replicate 2} & Het, Homo & Injection 1 & 139 & - \\
\hline & & Injection $2^{+}$ & 143 & 4 \\
\hline & & Injection 3 & 150 & 7 \\
\hline & & Sacrifice & 153 & 3 \\
\hline
\end{tabular}

Single littermates that underwent the same experimental treatment were used in each replicate.

$\mathrm{WT}=$ wild-type, Het $=$ Bmall $^{+/}$, Homo $=$Bmall $^{-/}$

${ }^{+}$Mice transferred to constant darkness until sacrifice. 


\section{Figure Legends}

Figure 1. Incremental growth lines in rodent incisor dentine: concentric lines indicated with white arrows. A) Hematoxylin-stained, undecalcified transverse section from a mandibular incisor of a 23 day old wild-type mouse (C57BL/6J background): En= enamel, De= dentine, $\mathrm{Am}=$ ameloblasts, $\mathrm{Od}=$ odontoblasts. $\mathrm{B}$ ) Right mandible of a mouse, showing the section plane for panel A and Figures $3-8$. Sections were cut in approximately the same region below the first molar as this includes dentine deposited during the duration of the experiment. C) Polarized light image of hematoxylin-stained, decalcified section from the tip of a rat incisor. (D) Polarized light image of unstained, undecalcified section from the tip of a rat incisor. Scale bar in A, C, D = 200 microns, $\mathrm{B}=2 \mathrm{~mm}$.

Figure 2. Elemental image of lead $(\mathrm{Pb})$ in a longitudinal section of an undecalcified maxillary incisor and surrounding bone from a wild-type mouse injected three times with Pb-NTA. Color scale is in parts per million.

Figure 3. Transverse sections of rat incisors cut below the first molar from each treatment group of the SCN lesion study. A, E) Decalcified section showing three lead labels; their clarity varied due to subtle planar obliquity. B, F) Undecalcified incisor from an untreated control rat. C, G) Undecalcified incisor from a sham surgery rat. D, H) Undecalcified incisor from a rat with a SCN lesion. Note the strong circumferential lines in the thick undecalcified sections shown in panels $B-D$ (white arrows in magnified images $F-H)$. Lead labels are not evident in $C, D, G$ and $\mathrm{H}$, although these individuals were subject to the same injection schedule as the individual in A and E. Scale bar in $A-D=500$ microns, $E-H=100$ microns. 
Figure 4. Incremental lines in decalcified sections from rats subject to various treatment conditions. A) Untreated control rat. Note the wide horizontal light/dark banding pattern near top of the section (black arrows) and the finer horizontal lines in the middle to bottom of the section (white arrows). It was not possible to confirm the timing of incremental lines as lead labels were not administered to this individual. B) Sham surgery rat showing $\sim 14$ closely-spaced growth lines (white arrows) between labels 2 and 3. C) Sham surgery rat showing $\sim 7-8$ widely-spaced increments (black arrows) between labels 2 and 3. A faint approximately circadian pattern continues between label 3 and sacrifice. Sham surgery rats in B and C were subject to the same treatment protocol: injection 1; 5 days later - injection 2, sham surgery, and transfer to constant light; 7 days later - injection 3; 4 days later - sacrifice. Scale bar in A and $\mathrm{C}=100$ microns, $\mathrm{B}=$ 200 microns.

Figure 5. Decalcified section of a rat subject to lesion posterior to the SCN showing increasingly finer incremental rhythms ranging from daily ( 5 lines over 5 days: labels $1-2)$ to subdaily $(\sim 13$ lines over 7 days: labels $2-3$, followed by $\sim 11-12$ lines over 4 days: label 3 to sacrifice). Scale bar $=100$ microns

Figure 6. Decalcified section of a rat subject to a complete SCN lesion showing daily lines (white arrows). A) Overview of region (black box) shown below photographed B) in focus and C) out of focus. Panel C shows 5 daily lines between labels $1-2,7$ daily lines between labels 2 - 3, and 4 daily lines between label 3 and sacrifice. Scale bar in $\mathrm{A}=500$ microns, $\mathrm{B}$ and $\mathrm{C}=$ 200 microns. 
Figure 7. Subdaily incremental lines in decalcified sections from rats with complete SCN lesions. A) 10 - 12 lines (white arrows) between label 3 and sacrifice (4 days apart). B) $12-$ 14 lines between the surgery/label 2 and label 3 (7 days apart). Scale bar in A and B = 100 microns.

Figure 8. Daily growth lines in decalcified sections from wildtype, heterozygous and homozygous Bmal1 mice from replicate 1. Numbers and dotted white lines denote lead labels every 4 days, followed by sacrifice 4 days later. Mice were transferred from 12:12 light/dark cycle into constant darkness after injection 2. A) Bmal1 $^{+/+}$mouse, B) Bmal1 ${ }^{+/-}$mouse, C) Bmal1 ${ }^{-}$ / mouse. Scale bar in $\mathrm{A}=200$ microns, $\mathrm{B}$ and $\mathrm{C}=100$ microns.

Supplemental Figure 1. Coronal plates of the rat brain depicting the locations of SCN lesions made in the present study as shown by the black outlined and shaded areas in the ventral midline of each slice. Lesions represent a composite of 3 bilaterally-lesioned rats and 2 unilaterallesioned rats superimposed on anterior to posterior planes of the SCN from $-0.48 \mathrm{~mm}$ to -0.96 $\mathrm{mm}$ relative to Bregma. This illustration does not include the single rat that received a lesion posterior to the SCN. Plates adapted from Paxinos and Watson (2006). 


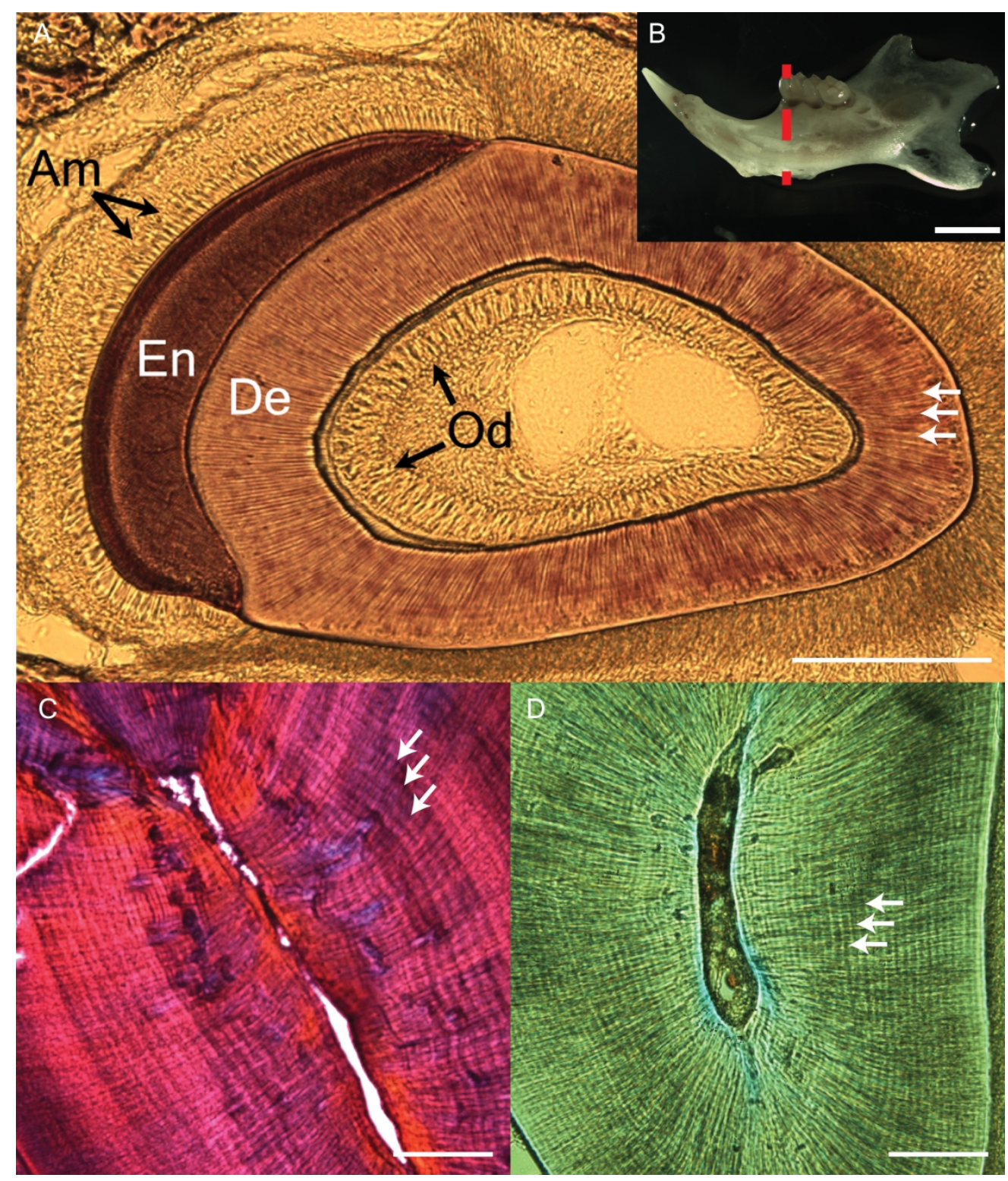

Figure 1 


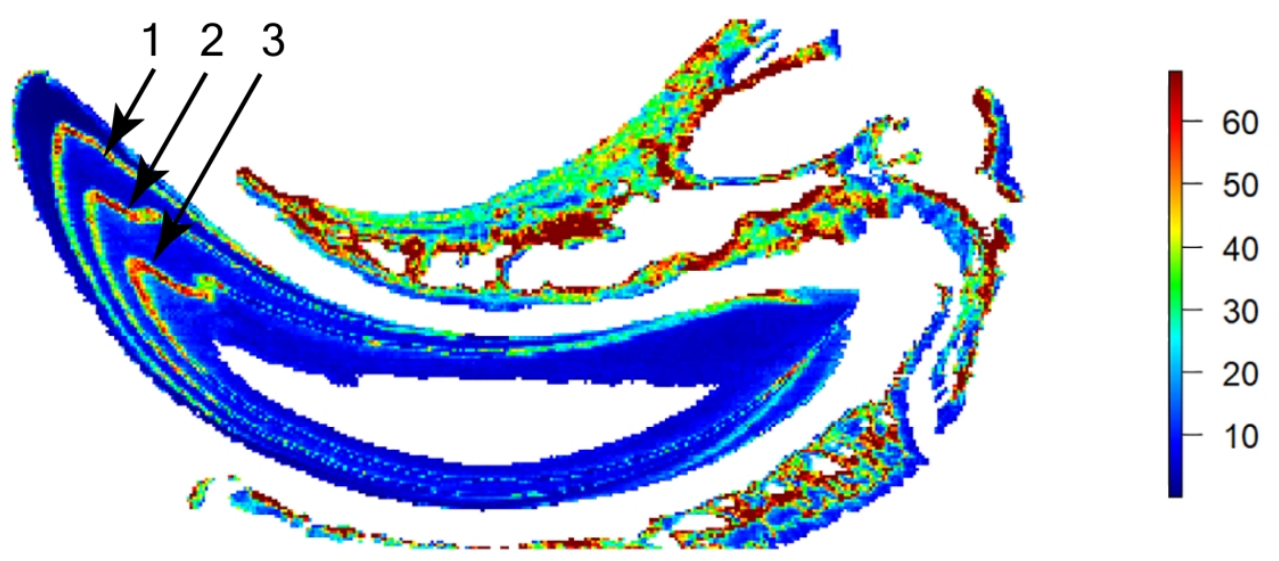

Figure 2 


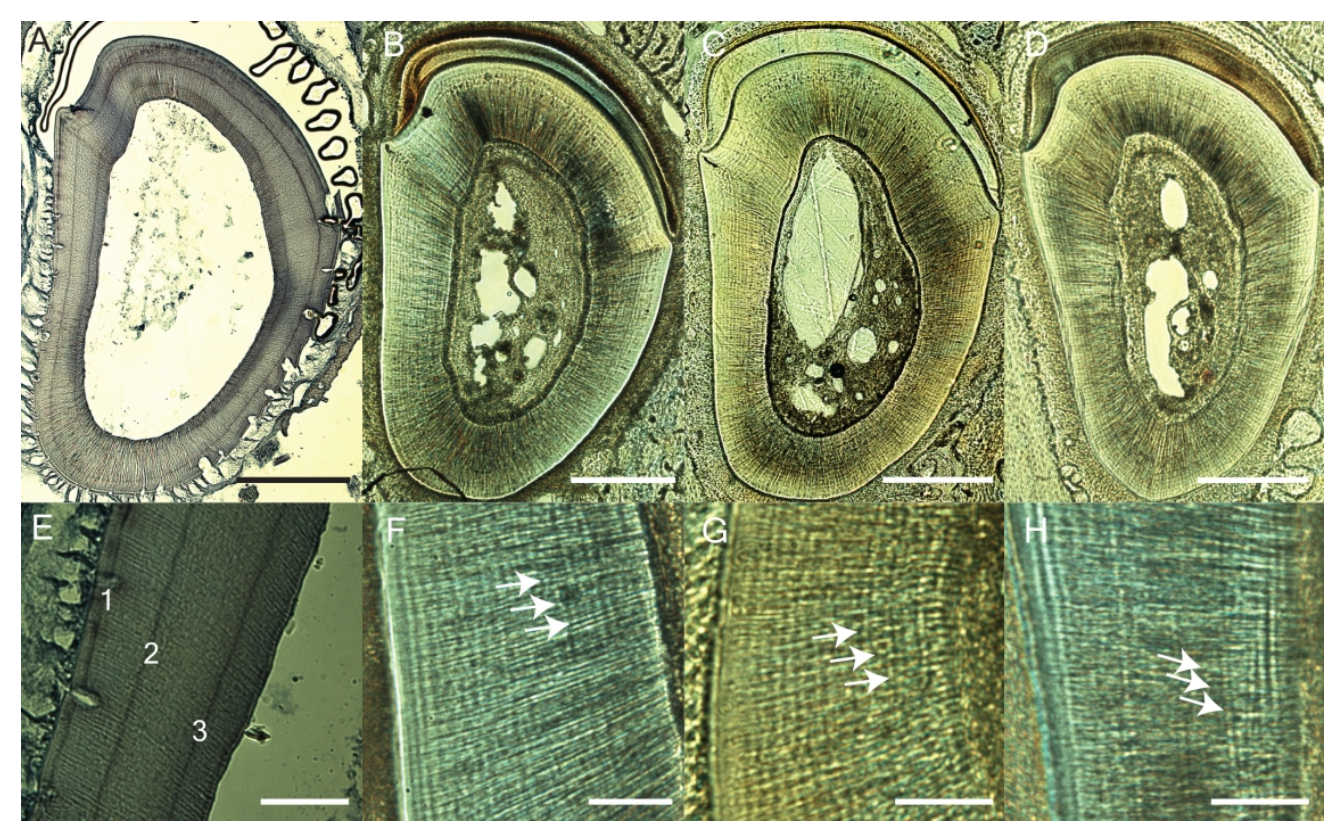

Figure 3 


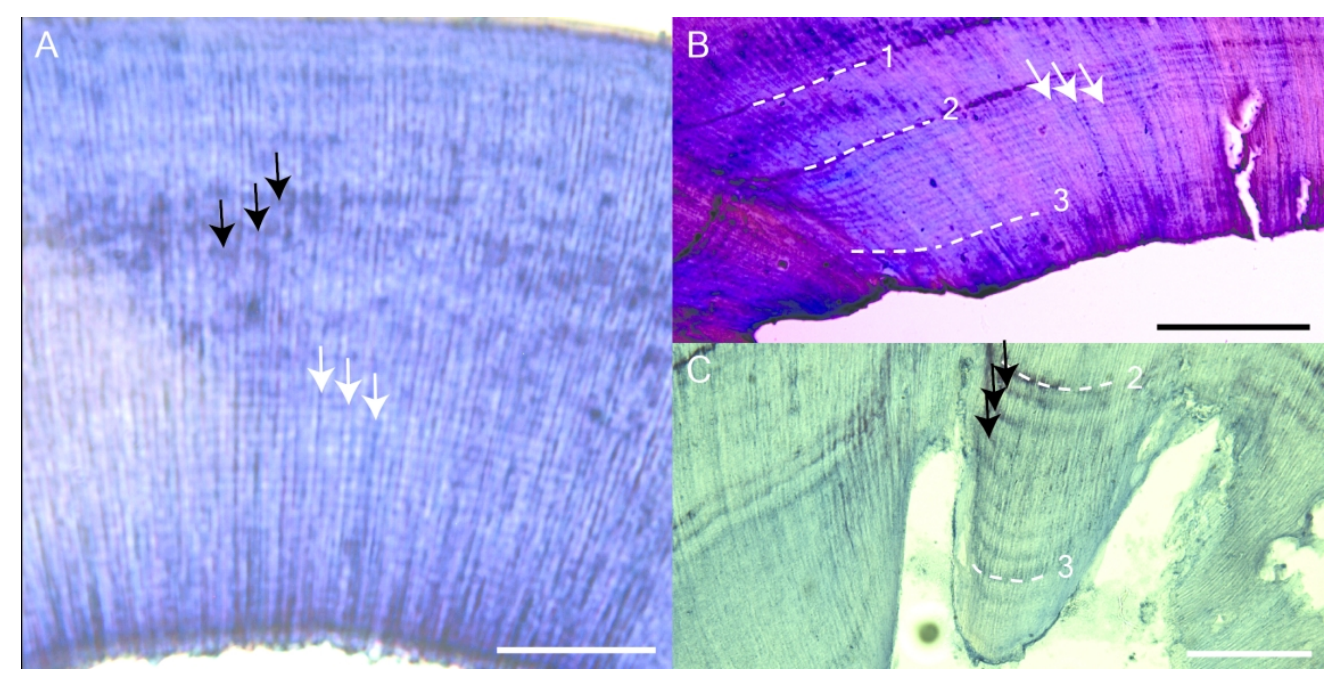

Figure 4 


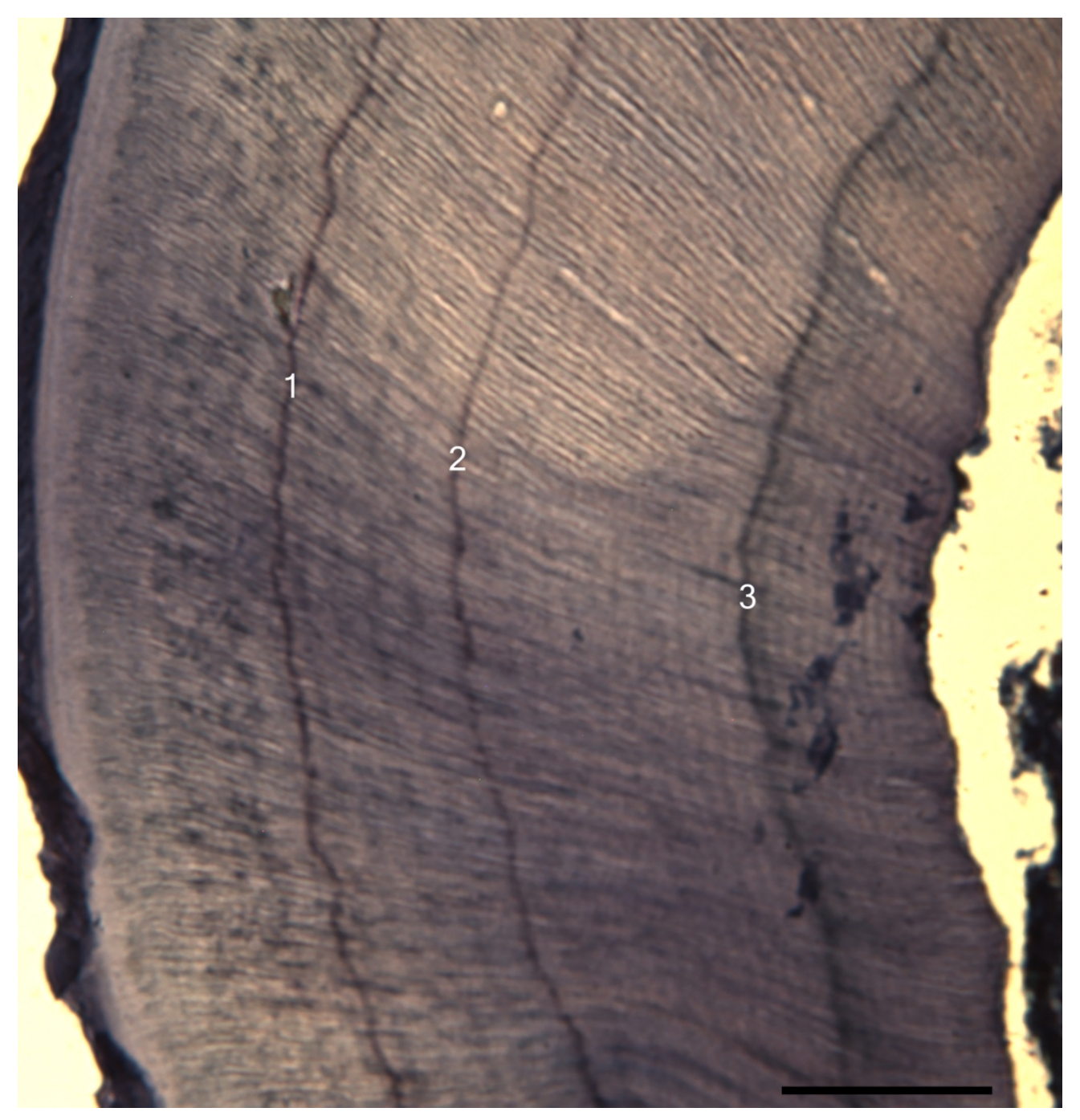

Figure 5 


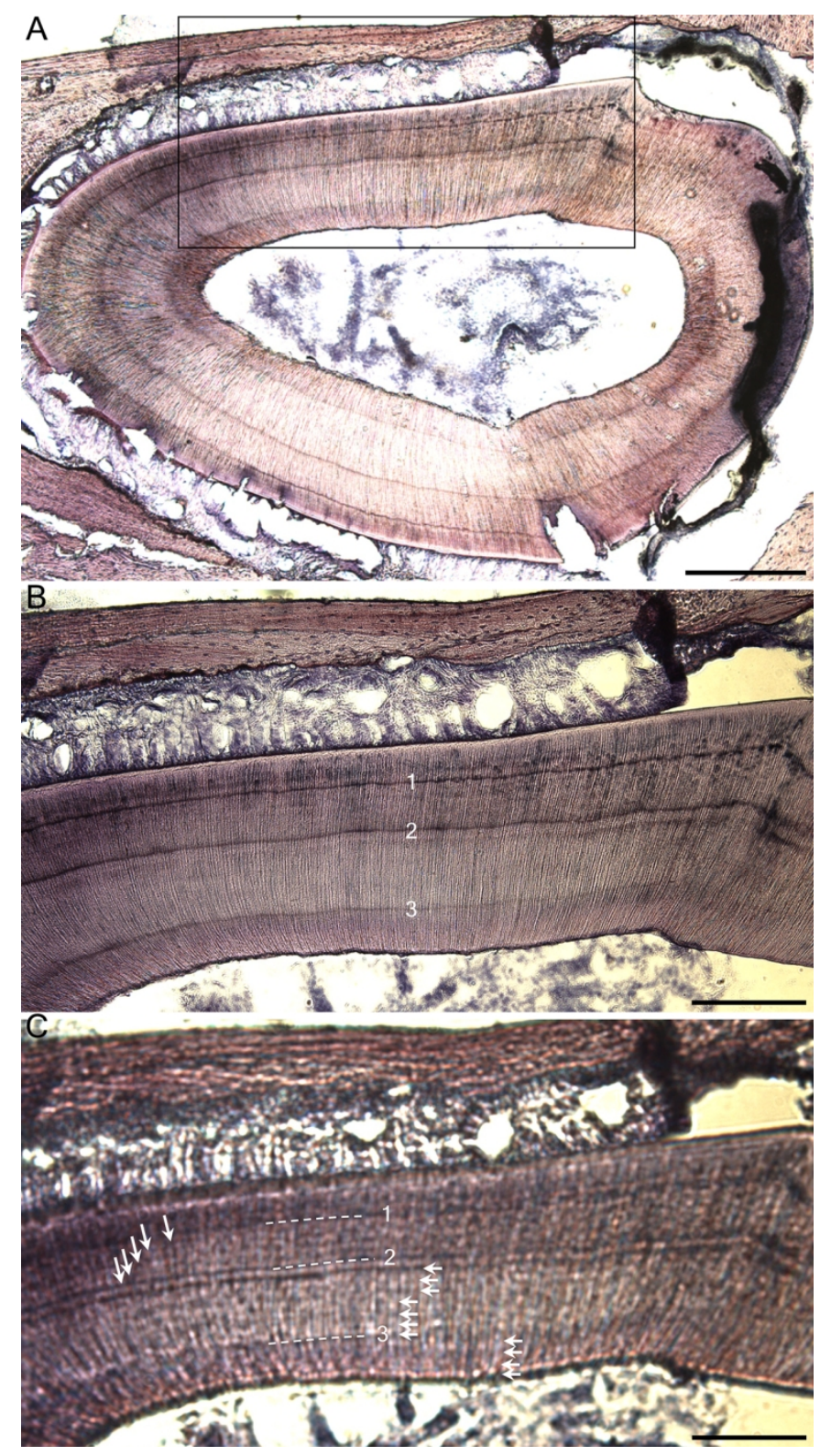

Figure 6

$101 \times 183 \mathrm{~mm}(300 \times 300$ DPI) 


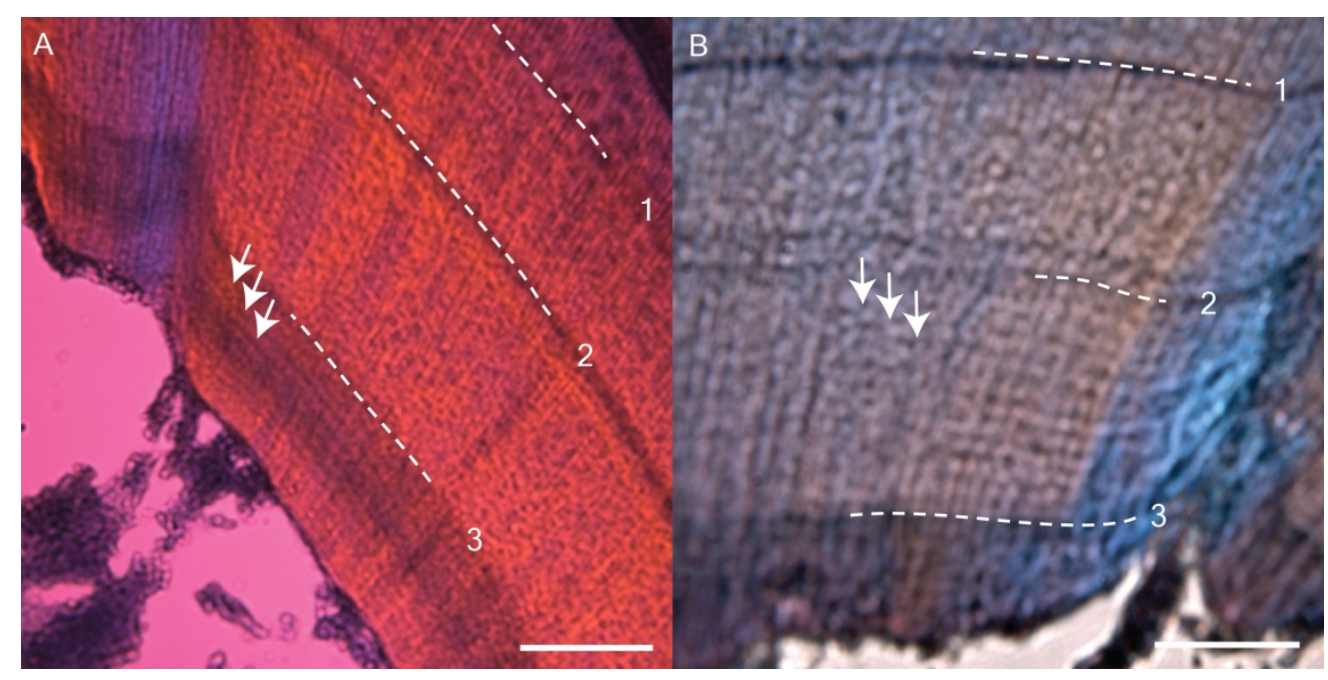

Figure 7 


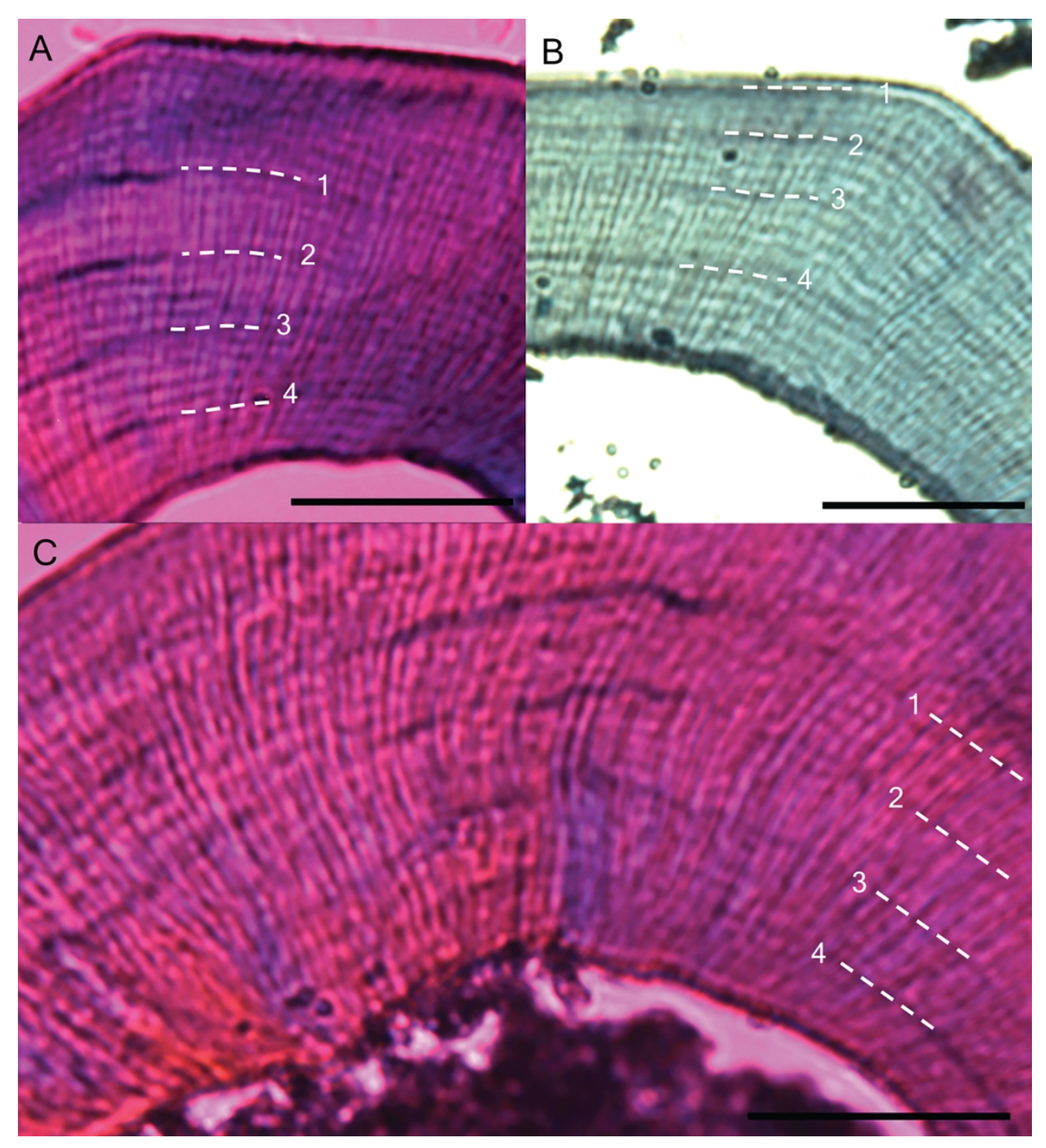

Figure 8 


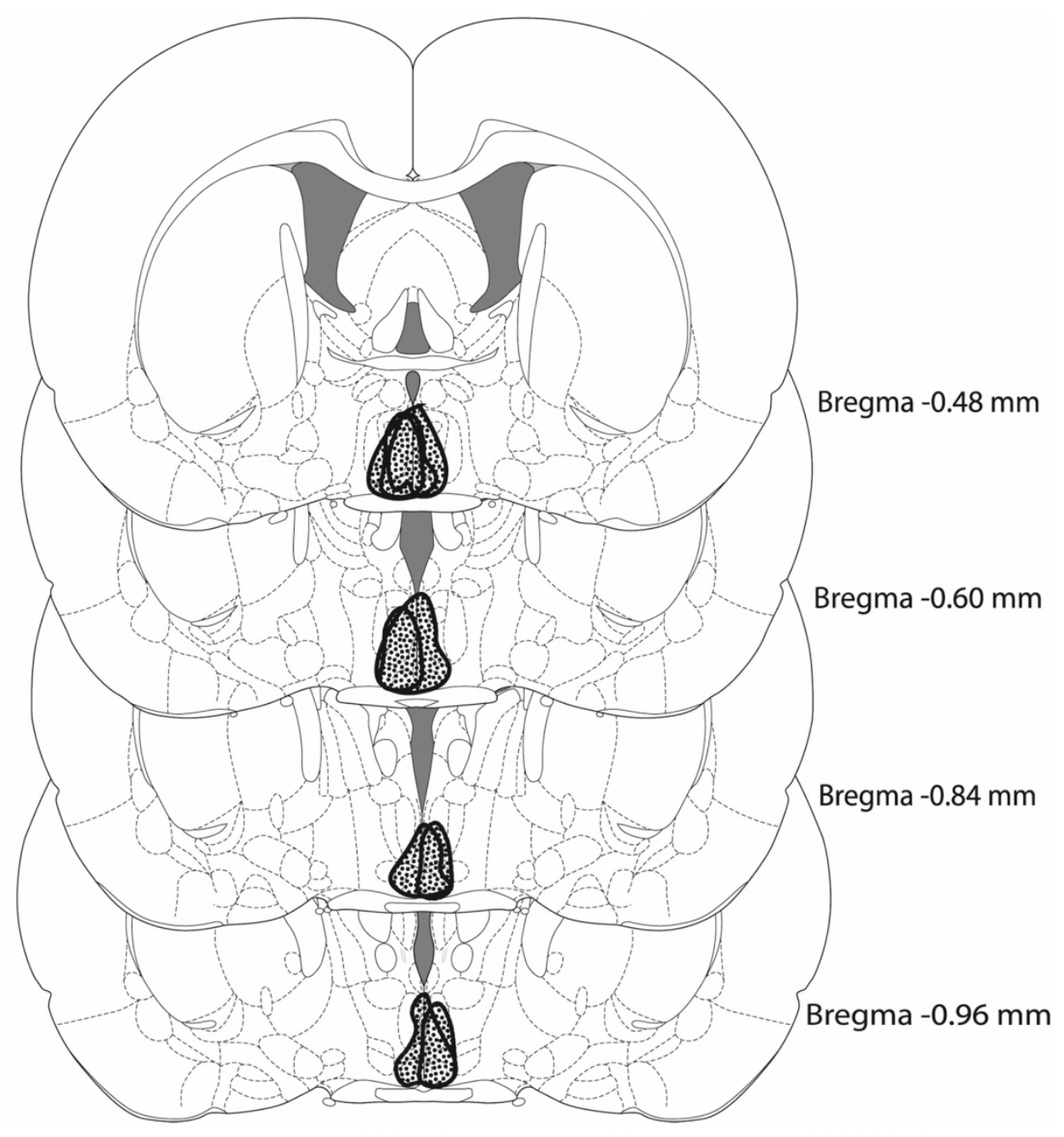

